\title{
Aproximación metodológica al impacto de WhatsApp y Telegram en las redacciones
}

\author{
Jon Sedano Amundarain \\ Universidad de Málaga \\ jon@uma.es \\ https://orcid.org/0000-0003-1845-7570
}

\author{
Ma Bella Palomo Torres \\ Universidad de Málaga \\ bellapalomo@uma.es \\ http://orcid.org/0000-0003-3100-3610
}

\section{Methodological approach to the impact of WhatsApp and Telegram in newsrooms}

\begin{abstract}
RESUMEN ABSTRACT
La inmediatez que exige Internet a los medios de comunicación ha provocado que muchas redacciones tengan que adaptar sus estrategias a nuevos formatos digitales (Andueza y Pérez, 2014). El auge de plataformas de mensajería como WhatsApp o Telegram ha propiciado que las redacciones comiencen a utilizarlas como canales de distribución para mantener informados a sus lectores (Batra, 2016). Las posibilidades multimedia que albergan, permitiendo enviar no solo texto y enlaces, sino también imágenes, vídeos y audios, ha propiciado el crecimiento exponencial de su implantación. La mayoría de medios se decantan solo por la emisión, mientras que una minoría opta por un uso centrado solo en la recepción de mensajes. Los estudios realizados hasta el momento contemplan una diversidad de estrategias por parte de los medios que buscan convertir las plataformas de mensajería en un nuevo canal de información (Negreira, López y Lozano, 2017). El objetivo principal de esta investigación consiste en recopilar y examinar los enfoques metodológicos aplicados hasta la fecha al estudio de la

The immediacy that Internet requires of mass media has led many newsrooms to adapt their strategies to new digital formats (Andueza and Pérez, 2014). The rise of messaging platforms such as WhatsApp or Telegram has led the newsrooms to begin using them as distribution channels to keep their readers informed (Batra, 2016). The multimedia possibilities they host, allowing not only text and links to be sent, but also images, videos and audios, have led to the exponential growth of their implementation. Most media opted only for the broadcast, while a minority opted for a use focused only on receiving messages. The studies carried out so far contemplate a diversity of strategies by the media that seek to turn messaging platforms into a new information channel (Negreira, Lopez and Lozano, 2017). The main objective of this research aims to compile and examine the methodological approaches applied to the study of the immediate Communications from an international approach, and to propose a specific tool that allows analyzing these messages peculiarities.
\end{abstract} comunicación inmediata desde una perspectiva internacional, y proponer una herramienta propia que permita analizar dicha actividad, y especialmente las particularidades de los mensajes publicados.

PALABRAS CLAVE

WhatsApp, Telegram, Mensajería inmediata, Periodismo móvil, Medios online

\section{KEYWORDS}

WhatsApp, Telegram, Instant messaging,

Mobile journalism, Online media 


\section{Introducción}

La saturación de contenidos en Internet y la distracción que generan los móviles han exigido que los periodistas realicen un esfuerzo extra para desarrollar nuevas estrategias que faciliten que su trabajo llegue a los lectores y se adapte a un escenario de cambios constantes (Catalina-García, García y Montes, 2015). Implicar a la audiencia de forma activa en un periodismo colaborativo, experimentando e introduciéndola en el proceso de gatekeeping, es un reto pero también una oportunidad para el crecimiento y la fidelización de los públicos (Bilton, 2014).

En los medios tradicionales, las historias mueren cuando son publicadas. En los entornos digitales publicar puede ser el comienzo de una gran historia, que se nutre de los inputs que llegan de fuentes espontáneas que voluntariamente desean participar en el diseño más completo de la pieza informativa. En este sentido, la audiencia condiciona el contenido (Batra, 2016).

El uso de aplicaciones de mensajería como WhatsApp o Telegram ha supuesto cambios radicales en la actividad interna y externa de las redacciones, al transformar rutinas y la manera de transmitir las noticias (Andueza y Pérez, 2014). Algunos medios disponen de un número asociado para comunicarse con los usuarios, distribuir contenidos y/o recibir tanto feedback como informaciones que son utilizadas como fuentes. Otra de las funciones atribuidas a estos servicios de mensajería instantánea consiste en potenciar la comunicación interna entre el equipo de periodistas.

"Modelamos nuestras herramientas y estas nos modelan a nosotros", explicaba Herbert Marshall McLuhan. La célebre frase "el medio es el mensaje" sigue siendo un referente para todo analista de medios de comunicación. Por esta razón, y debido a la expansión y al uso intensivo de las aplicaciones de mensajería en la sociedad actual, es necesario analizar cómo se comportan los medios de comunicación en estas plataformas (Bianda, 2014). Investigar sus peculiaridades permite saber el tipo de mensajes que se envían, su forma y las herramientas que utilizan para gestionar este contenido y hacer un seguimiento de su audiencia.

\section{Evolución del uso periodístico de WhatsApp}

Aunque WhatsApp se lanzó en 2009, y poco después surgieron algunos estudios iniciales como el de Mare (2013), su uso a nivel periodístico no empezó a ser notable hasta 2014. Telegram necesitó un periodo de asentamiento de unos tres años antes de ser utilizado por los medios de manera masiva. Sus virtudes frente a WhatsApp le han hecho convertirse en una de las aplicaciones de comunicación instantánea más idóneas para el uso periodístico (Quiroz, 2015).
Trushar Barot y Eytan Oren (2015) aseguran que las aplicaciones de mensajería tienen en la actualidad más usuarios globales que las redes sociales tradicionales porque permiten actuar con datos demográficos nuevos o difíciles de conseguir y que de cara a los testigos oculares de hechos son una plataforma en la que se sienten más seguros que hablando por teléfono. Por este motivo, aunque las aplicaciones de comunicación inmediata nacieron como una evolución de los SMS con bajo coste, con el tiempo han logrado ofrecer posibilidades multimedia que son aprovechadas por los medios. Los pioneros fueron BuzzFedd, Mashable, The Huffington Post, Vice o BBC News (Albeanu, 2015).

Su expansión ha derivado en la creación de nuevos perfiles profesionales, como el de WhatsApp Manager, un gestor encargado de conocer al detalle cómo se ha de interactuar con los usuarios mediante la aplicación. Paul Bradshaw (2015) explica que además de elegir a un responsable de WhatsApp en la redacción (Renner, 2016), lo más idóneo para los medios es utilizar listas de difusión. Sin embargo, hay casos como el del Washington Post que hace uso del chat convencional de la aplicación para comunicarse de forma directa e individualizada con los lectores.

The Guardian en diciembre de 2015 realizó una cobertura en tiempo real del debate electoral de Estados Unidos. Joseph Lichterman (2015) recogió las declaraciones del editor del Mobile Innovation Lab, Sasha Koren: "El objetivo del experimento era ver cómo The Guardian podría cubrir un evento en una aplicación de chat y también ver cómo era utilizar WhatsApp como una plataforma de publicación".

En noviembre de 2014, la organización por los derechos de la comunicación On Our Radar y la BBC cubrieron las elecciones nigerianas gracias a 36 periodistas ciudadanos que enviaban SMS y mensajes multimedia vía WhatsApp al medio (Reid, 2015). Pero no solo los corresponsales autóctonos utilizan este tipo de aplicaciones para comunicarse con el medio a nivel internacional (O’Donovan, 2014). La cobertura de la revolución de los paraguas de Hong Kong fue realizada mediante aplicaciones de mensajería por corresponsales que se desplazaron al país (Agur, Belair-Gagnon y Frisch, 2016).

Las posibilidades multimedia han facilitado la aparición de proyectos como Politibot (Ciobanu, 2016), un robot para Telegram creado con motivo de las elecciones generales de España por un grupo de periodistas. Gracias a él, los usuarios podían estar informados en todo momento sobre las últimas noticias concernientes a este evento político.

WhatsApp ha impulsado el periodismo móvil (Holanda, 2016). Su auge (Zanottti, 2016) y el de Telegram han provocado que la elaboración de noticias, antes condicionada por el SEO, se caracterice por un nuevo formato que aproxime al usuario hacia el medio (Custódio y Pires, 2015). En algunos casos los mensajes cuentan con una narrativa diferente a la utilizada 
por el medio en su web, más cercana y directa, con emoticonos que sirven para tal fin (Sampietro, 2016).

A pesar de estos antecedentes y de la relevancia del fenómeno, desde la academia la literatura al respecto es escasa y reciente. Hasta el momento, los estudios han abordado el análisis de ciertas coberturas puntuales (Ramalho, 2014) o del uso que algunos medios concretos hacen de estas aplicaciones de mensajería (Cdeperiodismo, 2014). Pero no se había realizado un análisis exhaustivo de un amplio volumen de medios (Shoutern, 2014). Por este motivo, se optó por revisar los medios nacionales que hacen uso de este tipo de aplicaciones y analizar su comportamiento durante un periodo concreto de tiempo.

El único estudio con el que se comparte un enfoque similar fue el realizado por Negreira, López y Lozano (2017), donde se analiza el uso que cinco medios hacen de estas herramientas de comunicación inmediata.

\section{Metodología}

El estudio "Aproximación metodológica al impacto de Whatsapp y Telegram en las redacciones" examina los principales cibermedios nacionales y locales observando qué tipo de sistema de comunicación inmediata utilizan. Para su consecución, se ha diseñado un directorio y dos fichas de análisis cuantita- tivo y cualitativo. Estas herramientas permiten examinar con detalle las cuentas y obtener los datos de los que se extraerán las conclusiones.

\subsection{Determinación de la muestra}

El directorio inicial se componía de 123 medios y programas. En el primer repaso se localizaron otros 18 medios internacionales que hacían un uso continuo de WhatsApp para comunicarse con los usuarios y que permitían contextualizar el caso español.

Tras confeccionar la lista se creó una tabla en la que se rellenaron diferentes datos: nombre del medio, web principal, enlace al comunicado en el que se anuncia el uso de la aplicación de mensajería, fecha en la que da inicio el servicio, número de teléfono utilizado o cuenta, si aparece en portada de la web, el tipo de uso que le dan al servicio (emisor o receptor de información), el canal utilizado (WhatsApp o Telegram), si está habilitada la opción de compartir noticias en estas aplicaciones desde el propio medio y un apartado para notas.

El siguiente paso consistió en determinar qué medios estaban activos en la actualidad. Como el análisis se focalizó en la faceta emisora, se excluyeron los que solo tenían un número de teléfono asociado a WhatsApp para que los usuarios enviaran contenido. Con los restantes se pasó a solicitar el alta en

\begin{tabular}{|c|c|c|c|c|}
\hline MEDIO & WEB PRINCIPAL & ENLACE & FECHA & NÓMERO \\
\hline 97.7 Radio & hitter/luma la977 com & & & 627980336 \\
\hline$A B C$ & htted/lumw abces & & & \\
\hline Antena 3 & bites//umw antena $3.00 m$ & & & \\
\hline As & http:llas.com & htto://futbol.as com/futbol/2015/03/18/primeral: & $19 / 03 / 2015$ & 667350009 \\
\hline Cadena 100 & hitte:/lwown. cadena100.es & & & \\
\hline Cadena SER & httelicadenasercem & & & \\
\hline Cambio16 & bits $/ /$ /manc cambio $16 . \mathrm{com}$ & httes://unww facoboek com/Cambio $16 /$ bosts/11493 & $12 / 04 / 2016$ & @cambio16 \\
\hline Canal Sur & bite //inmw.canalsucesl & & & \\
\hline Canal Sur (Gente maravillosa) & hittes/imow.canalsuces/ & bitte//homw can-alsures/television/pregramas/gents & $11 / 01 / 2017$ & 664575289 \\
\hline Canarias 7 & httes//mow canarias 7 es & & & \\
\hline Catalunya Radio & htte-liseatalunyaradio cat & & & \\
\hline Cinco Dias & htte/licincodias com & & & \\
\hline COPE & $\mathrm{bite} / \mathrm{hmon}$. cose.es & & & \\
\hline CTX & bitteslictoties & & $16 / 03 / 2016$ & Qrevistactx \\
\hline Cuarto Poder & hittes:/unww cuartopoderes & 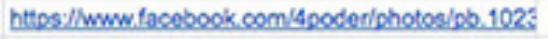 & $12 / 05 / 2016$ & QCuartoPoder \\
\hline Diari de Girena & httes//wnw diaridegircona ceat & & & \\
\hline Diario Aragonés & $\mathrm{http} / \mathrm{hmw}$ diarioaragenes.cem & & & \\
\hline Diario de Almeria & bitos/letalmeria.es & & & \\
\hline Diario de Cadiz & htteylidiariodecadizcom & & & \\
\hline Diario de Ibiza & htto //Mnow diariedeibiza es & & & \\
\hline Diario de Jerez & htte $/ /$ manw diariodojeroz es & & & \\
\hline Diario de León & 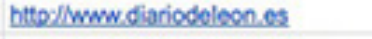 & & & \\
\hline Diario de Malorca & bites/lumenciariodemallorea.es & 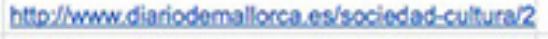 & $09 / 06 / 2016$ & 619531163 \\
\hline Diario de Navarra & bitte/limmw diariodenavarra.es & htto://monw diariodenavarra es/nosiclas/navarra/20 & $05 / 03 / 2017$ & 687040098 \\
\hline Diario de Sevilla & htted/mow diariodesovilla es & & & \\
\hline Diario del AltoAragón & htte///wnw diariodelaltoaragen os & & & \\
\hline Diario Información & bttes/lumw diarioinformacion.cem & & & \\
\hline Dario Motor & hitto/lunw diariomelor.com & 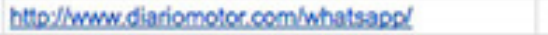 & $11 / 09 / 2015$ & 644290756 \\
\hline
\end{tabular}

Figura 1. Extracto del directorio de medios españoles que sirve para determinar si utilizan WhatsApp o Telegram (2017). Elaboración propia. 


\begin{tabular}{|l|l|l|l|l|l|}
\hline Medio & Web principal & Enlace & Fecha & Número & Anunciado en portada \\
\hline SUR & http://sur.es & $\begin{array}{l}\text { http://www.diario- } \\
\text { sur.es/tecnologia/ }\end{array}$ & $530 / 08 / 2016$ & 660481739 & Sí \\
\hline
\end{tabular}

Tabla 1. Ejemplo de la tabla de análisis inicial (Parte A). Elaboración propia

\begin{tabular}{|l|l|l|l|l|}
\hline Uso & Canales & Opción de compartir & Notas & Forma de darse de alta \\
\hline Emisor / Receptor & WhatsApp y Telegram & No & Suscrito & $\begin{array}{l}\text { Enviar "ALTA + nombre" o suscribirse a canal } \\
\text { de Telegram }\end{array}$ \\
\hline
\end{tabular}

Tabla 2. Ejemplo de la tabla de análisis inicial (Parte B). Elaboración propia

todos sus canales o listas de difusión, descubriendo que algunos ya no estaban en vigor, ya sea porque nunca llegaron a contestar o porque el número estaba asociado a un particular que nada tenía que ver con el medio.

La lista final de medios nacionales que hacen uso como emisores de WhatsApp o Telegram se redujo a 27 medios, de los cuales 4 utilizaban los dos sistemas para compartir información.

\subsection{Fichas de análisis}

Mediante el cuestionario inicial se recopilaron los principales datos de las cuentas con las que se crearon las fichas de análisis. Con estas fichas se obtuvieron resultados cuantitativos (tamaño de sus canales, número de actualizaciones diarias, tipología de los mensajes en función del uso de texto, imagen o vídeo, volumen de emoticonos utilizados, etc.) y cualitativos (calidad de las respuestas a preguntas preestablecidas, tipología de lenguaje o de las informaciones compartidas). Debido a las diferencias entre WhatsApp y Telegram, se crearon dos tipos diferentes de fichas, cada una orientada a una aplicación. Aun así, como las dos guardan estrecha relación, varios de los ítems a estudiar son coincidentes.

Las fichas están divididas en tres bloques: Plataforma analizada (junto al nombre de la app para determinar el tipo de ficha), Análisis de la cuenta y Análisis del contenido. La primera y segunda parte solo requieren que se cumplimenten una vez, salvo la fecha de análisis, mientras que la tercera varía de forma completa en función del día analizado.

\subsubsection{Plataforma analizada}

Tanto en Telegram como en WhatsApp se incluyen dos parámetros iguales: Fecha de análisis, para indicar el día al que corresponde, y Fecha de inicio del servicio, donde se indica cuándo comenzó el medio a utilizar el servicio. En el caso de WhatsApp se añade: Forma de darse de alta, ya que algunos requerían enviar la palabra "Alta", otros en cambio pedían poner el nombre, etc.

\subsubsection{Análisis de la cuenta}

Respecto a los datos de las cuentas, tanto el punto número uno como el dos, están dispuestos para marcar afirmativa o negativamente la respuesta correcta. El ítem 1 se refiere a si el servicio está anunciado en la portada de la web. El ítem 2 se centra en documentar si el medio utiliza un logo personalizado para el chat.

Los ítem 3 y 4 varían dependiendo de la aplicación. En WhatsApp, el apartado 3 indica el número de teléfono, mientras que el 4 se anota el tiempo de reacción del medio a la hora de dar

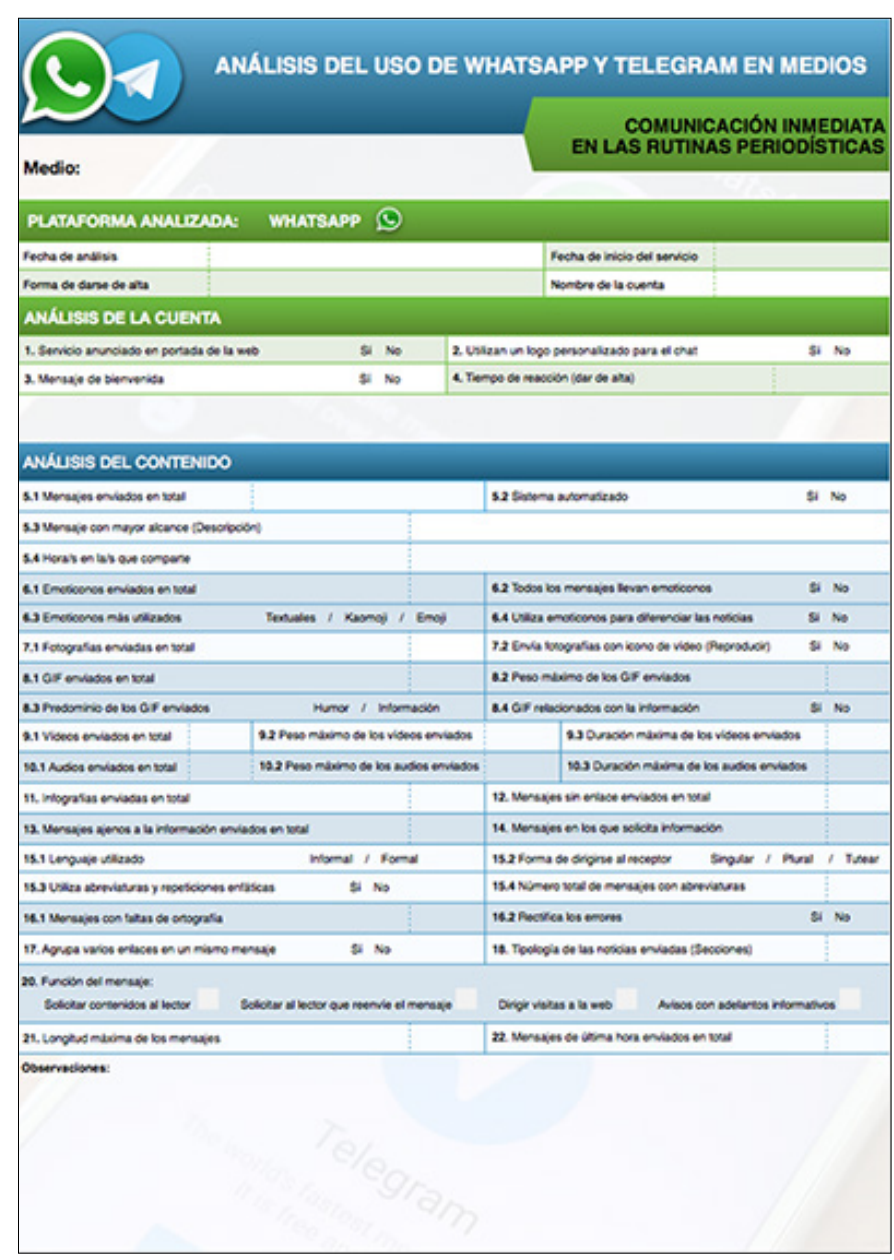

Figura 2. Ficha diseñada para analizar la actividad de un medio en WhatsApp. Elaboración propia. 


\section{PLATAForma analizada: telegram}

\section{Fecha de análisis}

Forma de darse de atto

Fecha de inicio del servic

Nombre de la cuenta

Figura 3. Primera parte de la ficha correspondiente a Telegram. Elaboración propia.

de alta a un usuario en su lista de difusión. En Telegram en cambio, el punto 3 está reservado para poner el nombre de la cuenta y el 4 para indicar el número de seguidores que tiene el canal del medio en la app.

\section{ANÁLISIS DE LA CUENTA}

\begin{tabular}{|c|c|c|c|}
\hline nciado en portada de la web & Si No & 2. Utilizan un logo personalizado para el chat & Si No \\
\hline 3. Mensaie de bienverida & Si No & 4. Nümero de ss & \\
\hline
\end{tabular}

Figura 4. Segunda parte de la ficha correspondiente a Telegram. Elaboración propia.

\subsubsection{Análisis de contenido}

Representa el área más extensa de la ficha. Dentro del punto 5 se establecen los datos referentes a los mensajes en sí: Mensajes enviados en total durante el día, si hacen uso de un sistema automatizado y por lo tanto los mensajes se reducen a compartir la noticia sin textos adicionales y las horas en las que han compartido los mensajes. En Telegram se amplía este punto con el apartado Mensaje con mayor alcance, donde se deja un campo abierto para explicar su contenido. Los puntos bajo este epígrafe sirven para tener un baremo del número de mensajes medio que envían al día, así como las principales horas y el contenido más exitoso en el caso de Telegram.

El epígrafe 6 es similar para ambas aplicaciones y se centra en el uso que el medio hace de los emoticonos en sus mensajes. Determina el número de emoticonos enviados en total, si todos los mensajes incluyen algún tipo de emoticono, cuáles son los más utilizados (textuales, kaomoji o emoji) y si los utiliza para diferenciar las noticias. Los emoticonos se han convertido en algo habitual en las comunicaciones digitales informales. Aunque los medios tradicionales no hagan uso de ellos en sus textos de forma habitual, en este tipo de aplicaciones de mensajería es frecuente su aplicación, incluso por parte de los medios. De ahí la necesidad de analizar en profundidad su presencia.

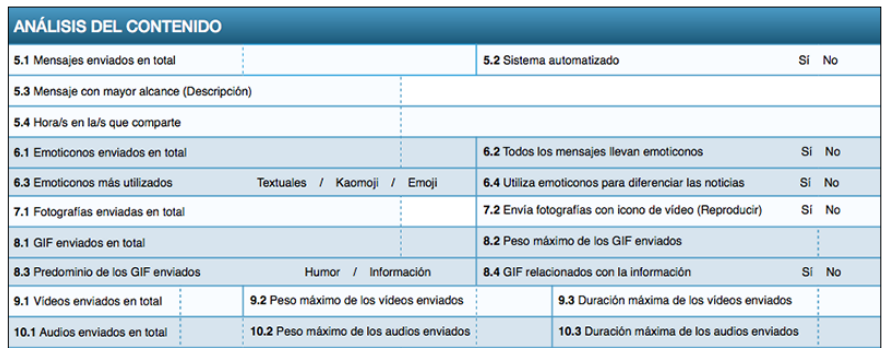

Figura 5. Tercera parte de la ficha correspondiente a Telegram. Elaboración propia.
El siguiente apartado, el 7, se centra en la fotografía con dos puntos a cumplimentar: el número de fotografías enviadas en total y si estas se remiten con el icono de un vídeo. Este último punto se incluyó al percibir que algunos medios enviaban así enlaces de vídeo con el objetivo de incentivar a los usuarios a hacer clic en ellos.

Le sigue el análisis de los GlF. En ambos casos se marca el número total de GlF enviados, el peso máximo de estos teniendo en cuenta el gasto de datos para los usuarios, el tipo de GIF predominante (humor o información) y si están relacionados con la información que se está ofreciendo. Los puntos 9 y 10 tienen una estructura similar. El primero centrado en vídeo y el segundo en audios. En los dos puntos se establece el número total que se ha enviado, el peso máximo, de nuevo para ver si tienen en cuenta el consumo de los usuarios y la duración máxima.

El número total de infografías enviadas (punto 11) y el número total de gráficas enviadas (punto 12) son los siguientes epígrafes que aparecen en la ficha de análisis, con objeto de conocer el protagonismo que poseen estos contenidos visuales en la comunicación inmediata. Les sigue un apartado donde se analiza si se envía algún tipo de contenido ajeno a la información y el número total de mensajes sin enlace enviados.

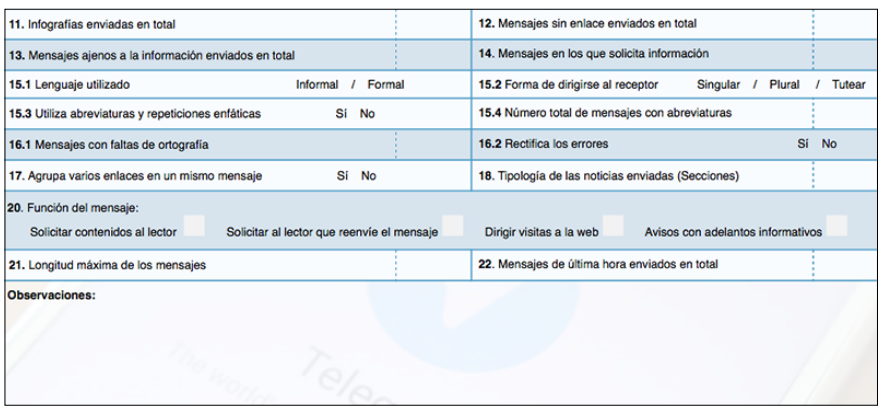

Figura 6. Parte final de la ficha correspondiente a Telegram. Elaboración propia.

El apartado 15 está más centrado en el análisis cualitativo. El primer punto determina el tipo de lenguaje utilizado, si es informal o formal. En el punto 2 se analiza la forma en la que el medio se dirige al receptor: singular, plural o tuteo. El uso de abreviaturas o repeticiones enfáticas se analizan en el tercer punto. Un último punto analiza el número total de mensajes con abreviaturas.

Después se analiza el volumen de mensajes con faltas de ortografía (punto 16.1) y si rectifican los errores (16.2), ya sea mediante un mensaje adicional o corrigiendo el mensaje enviado, algo que permite Telegram y que recientemente ha incorporado WhatsApp.

El punto 17 está centrado en ver si envían mensajes recopilatorios, con varios enlaces y el 18 en el número de mensajes en los que se solicita información al usuario. Aunque los medios 
analizados ejerzan principalmente como emisores, en ocasiones puntuales puede que soliciten algún tipo de colaboración por parte de los usuarios dirigiéndoles mediante un enlace o texto a otro lugar (formulario en página web, correo o número de contacto).

En el punto 19 se indica la tipología de las noticias enviadas, cumplimentando la zona del apartado con las secciones del medio a las que pertenecen. Y el punto 20 se centra en las funciones, dividiéndose en cuatro opciones: solicitar contenidos al lector; solicitar al lector que reenvíe el mensaje; dar visitas a la web y avisos con adelantos informativos.

Los dos últimos ítems abordan la longitud máxima de los mensajes (punto 21), estableciendo el número de caracteres del mensaje más largo enviado ese día, y los mensajes de última hora enviados (punto 22).

Por último, se dejó un espacio final con la casilla Observaciones con el objetivo de escribir contenido adicional que se crea oportuno señalar para un análisis más completo.

\begin{tabular}{|c|c|}
\hline Medio & Plataforma \\
\hline Cambio 16 & Telegram \\
\hline CTX & Telegram \\
\hline Cuarto Poder & Telegram \\
\hline Diario de Mallorca & WhatsApp \\
\hline Diario de Navarra & WhatsApp \\
\hline EFE & Telegram \\
\hline El Comercio & WhatsApp \\
\hline El Español & Telegram \\
\hline El Huffington Post & Telegram \\
\hline El País & Telegram \\
\hline El Periódico & Telegram / WhatsApp \\
\hline ElDiario.es & Telegram / WhatsApp \\
\hline Faro de Vigo & WhatsApp \\
\hline La Casa de EL & Telegram \\
\hline La Nueva España & WhatsApp \\
\hline Las Provincias & Telegram / WhatsApp \\
\hline Levante-EMV & WhatsApp \\
\hline Maldito Bulo (La Sexta) & Telegram \\
\hline Mallorca Zeitung & WhatsApp \\
\hline Microsiervos & Telegram \\
\hline OK Diario & Telegram \\
\hline Público & Telegram \\
\hline Público (Tremending) & Telegram \\
\hline SUR & Telegram / WhatsApp \\
\hline Verne & Telegram \\
\hline Verne (El Tragabulos) & Telegram \\
\hline Voz Pópuli & Telegram \\
\hline Xataka & Telegram \\
\hline
\end{tabular}

Tabla 3. Medios analizados durante el pretest. Elaboración propia

\subsection{Pretest}

Las fichas se sometieron a dos pretest con los que se logró asentar una herramienta funcional. Se estableció el tiempo de análisis, desde el lunes 15 de mayo de 2017 hasta el domingo 21 de mayo del mismo año. Para obtener información de ese periodo, durante el pretest se solicitó el alta en los medios como usuario, tanto en las listas de difusión de WhatsApp como en el canal de Telegram.

La conclusión extraída del análisis completo permitiría conocer cómo se comportan los principales medios de comunicación españoles También se estimaría la evolución y el uso que los medios le otorgan de forma global a las herramientas de comunicación inmediata.

\section{Conclusiones}

La realización de un directorio de medios permitió filtrar la población de estudio de manera que se ajustara a los criterios de inclusión y exclusión. De esta forma se obtuvo una muestra más representativa de los medios que se pretendieron estudiar.

Al aplicar los pretest en las fichas, se pudo afinar el contenido de estas para ajustarlas mejor a los objetivos del estudio "Aproximación metodológica al impacto de Whatsapp y Telegram en las redacciones". Las fichas han resultado ser satisfactorias en modo que analizan los datos de manera eficaz, cómoda y detallada. En la actualidad se está procediendo al análisis de los datos obtenidos para su posterior publicación de los resultados.

Se espera que estas herramientas puedan ser utilizadas en estudios posteriores, como por ejemplo para analizar cómo se comportan los medios ante eventos socialmente importantes o en periodos de mayor longitud.

\section{Nota}

Esta investigación se enmarca en el proyecto nacional "La influencia de la audiencia en la innovación periodística: riesgos y oportunidades" (CSO2015-64955-C4-3-R MINECO/FEDER).

\section{Referencias}

Agur C., Belair-Gagnon, V., y Frisch N. (2016). How Foreign Correspondents Use Chat Apps to cover Political Unrest. Columbia Journalism Review. Recuperado de http://www.cjr.org/tow_center_reports/ foreign_correspondents_chat_apps_unrest.php

Albenau C. (2015). How BBC Have Your Say uses WhatsApp for newsgathering, Journalism.co.uk. Recuperado de https://www.journalism. co.uk/news/how-the-bbc-uses-whatsapp-for-newsgathering/s2/ a565490/ 
Andueza, M., y Pérez R. (2014). El móvil como herramienta para el perfil del nuevo periodista. Historia y Comunicación Social, 19,591602.

Barot T., y Oren E. (2015). Guide to Chat Apps. Tow Center For Digital Journalism. Recuperado de http://towcenter.org/research/guide-tochat-apps

Batra B. (2016). News Comunication Throught WhatsApp. International Journal of Informative \& Futuristic Research, 3(10), 3725-3733.

Belair-Gagnon, V., Agur C., y Frisch N. (2017). The Changing Physical and Social Environment of Newsgathering: A Case Study of Foreign Correspondents Using Chat Apps During Unrest. Social Media + Society, (January-March 2017), 1-10.

Bianda E. (2016). Le messaggerie istantanee, un nuovo territorio dell'informazione. Problemi dell'informazione. Año XLI, (3), 637-639.

Bilton R. (2014). WhatsApp emerges as big share driver for publishers. DigiDay UK. Recuperado de http://digiday.com/media/publishers-see-big-sharing-numbers-via-whatsapp/

Bradshaw P. (2015) Lessons on using WhatsApp for publishing - an election experience. Online Journalism Blog. Recuperado de https:// onlinejournalismblog.com/2015/05/19/tips-on-using-whatsapp-forpublishing-an-election-experience/

Catalina-García B., García A., y Montes M. (2015). Jóvenes y consumo de noticias a través de Internet y los medios sociales. Historia y Comunicación Social, 20(2), 601-619.

CDEPERIODISMO. (2014). Estos son los medios que apuestan por el uso de WhatsApp. Clases de Periodismo. Recuperado de http://www. clasesdeperiodismo.com/2014/09/20/estos-son-los-medios-queapuestan-por-el-uso-de-whatsapp/

Ciobanu M. (2016). More than 6,000 people are talking to Politibot about the upcoming elections in Spain. Journalism.co.uk. Recuperado de https://www.journalism.co.uk/news/thousands-of-people-aretalking-to-politibot-about-the-upcoming-elections/s2/a649186/

Custódio L., y Pires J.L. (2015). A participaçao do ouvinte no Jornal da CBN: uma análise sobre a utilizaçao do aplicativo WhatsApp na constrçao do noticiario. Ancora: Revista Latino-americana de Jornalismo, 2(1),116-137.

Evans B. (2012). WhatsApp - The Biggest Social Network You've Never Heard Of. Forbes. Recuperado de https://www.forbes.com/ sites/benedictevans/2012/10/19/whatsapp-the-biggest-social-network-youve-never-heard-of/\#273df1172eb8

Holanda A. (2016). Whatsapp no jornalismo móvel: um recorte da realidade de quatro veículos alagoanos. Ancora: Revista Latino-americana de Jornalismo, 3(2), 50- 67.

Lichterman J. (2015). What the Guardian learned from its first WhatsApp live chat. NiemanLab. Recuperado de http://www.niemanlab. org/2015/12/what-the-guardian-learned-from-its-first-whatsapplive-chat/

Mare A. (2013). New Media Technologies and Internal Newsroom Creativity in Mozambique. Digital Journalism 2(1), 12-28. doi: 10.1080/21670811.2013.850196

Negreira, MC., López, X., y Lozano L. (2017). Instant Messaging Networks as a New Channel to Spread the News: Use of WhatsApp and Telegram in the Spanish Online Media of Proximity. Recent Advances in Information Systems and Technologies. WorldCIST 2017. Advances in Intelligent Systems and Computing, 571, 64-72.
O'Donovan C. (2014). Around the world, media outlets and journalists are using chat apps to spread the news. NiemanLab. Recuperado de http://www.niemanlab.org/2014/06/around-the-world-media-outlets-and-journalists-are-using-chat-apps-to-spread-the-news/

Quiroz, Y.R. (2015). Aplicación de whatsapp en el periodismo digital y ciudadano. Hamut'ay. Revista Científica de la División de Investigación y Extensión Científica Tecnológica, 2(1), 71-83.

Ramalho, G. (2014). Brazilian newspaper Extra celebrates one year using WhatsApp to connect reporters and readers. Journalism in the Americas. Recuperado de https://knightcenter. utexas.edu/blog/00-15670-brazilian-newspaper-extra-celebrates-one-year-using-whatsapp-connect-reporters-and-rea

Renner, N. (2016). A new role in journalism: the digital fixer. Columbia Journalism Review. Recuperado de http://www.cjr.org/tow_center/ new_role_journalism_digital_fixer.php

Reid, A. (2015). Covering the nigerian election - with just SMS and WhatsApp. Journalism.co.uk. Recuperado de https://www.journalism. co.uk/news/how-citizen-journalists-covered-the-nigerian-election-on-mobile/s2/a564669/

Sampietro, A. (2016). Emoticonos y multimodalidad. El uso del pulgar hacia arriba en whatsapp. Aposta: Revista de ciencias sociales, (69), 271-295.

Shoutern, L. (2016). How four publishers are using WhatsApp. Digiday UK. Recuperado de http://digiday.com/social/four-publishers-using-whatsapp/

Zanotti C. (2016). WhatsApp e a publicizaçao de noticias na sociedade midiatizada. $3^{\circ}$ Congreso de Jornalismo para Dispositivos Móveis. Recuperado de https://www.youtube.com/watch?v=gl40M2qHCuw

\section{CV}

Jon Sedano. Graduado en Periodismo e investigador en la Universidad de Málaga. Especializado a nivel profesional en periodismo multimedia y nuevas narrativas. Autor de varios artículos relacionados con herramientas de comunicación inmediata y verificación de fuentes. Líneas de investigación: innovaciones periodísticas y veracidad en la red.

Bella Palomo. Catedrática y Directora del Departamento de Periodismo de la Universidad de Málaga. Especializada en las áreas de cibermedios, audiencias activas, social media y rutinas periodísticas, es autora de un centenar de artículos y libros sobre estos temas. Ha sido consultora de multimedia y comunicación en la Universitat Oberta de Catalunya, y profesora visitante en las universidades de Washington, Rutgers, Miami (EUA), Shangai (China) y Federal de Bahía (Brasil). Actualmente es IP del proyecto "La influencia de la audiencia en la innovación periodística: riesgos y oportunidades" (CSO201564955-(4-3-R MINECO/FEDER). 